\title{
PREPARATION AND CHARACTERISTICS OF A CHEMICALLY AMPLIFIED SILICONE-BASED NEGATIVE RESIST
}

Akinobu Tanaka, Hiroshi Ban, Jiro Nakamura and Yoshio Kawai

NTT LSI Laboratories, Morinosato, Atsugi-shi, Kanagawa, 243-01

The characteristics of a chemically amplified silicone-based negative resist, composed of alkalisoluble silicone polymer and an acid generator, have been studied. This polymer is obtained through a sol-gel reaction of a mixture of phenyltrimethoxysilane and 2(3,4-epoxycyclohexyl)ethyl trimethoxysilane and has numerous silanol groups that make it alkali-soluble. The resist's chemistry is based on the acid-catalyzed condensation of the silanol groups during post exposure baking. The CSNR includes silicon atoms in such abundance that it exhibits high resistance to oxygen reactive ion etching and can be used as a top imaging layer of a two-layer resist system. Using a $\mathrm{KrF}$ excimer laser stepper, $0.25-\mu \mathrm{m}$ lines-and-spaces patterns can be fabricated with a two-layer resist system. The sensitivity depends on the structures of acid generators ( $\left.\mathrm{AG}^{\prime} \mathrm{s}\right)$. A resist with triphenylsulfonium triflate showed the highest sensitivity in this study.

\section{Introduction}

Chemically amplified resists are the most promising ones for $\mathrm{KrF}$ excimer laser lithography because of their high transmissibility and high sensitivity at $248 \mathrm{~nm} .1$ )-3) However, due to their high transmissibility and non-bleachable absorbance characteristic, the standing wave effect becomes serious. 3 ) On the other hand, the narrow focal tolerance in optical lithography deteriorates patterning characteristics especially in sub-half-micron regions. Using multi-layer resist systems (MLR) in $\mathrm{KrF}$ excimer laser lithography can overcome these problems. Among the MLR systems, two-layer resist (2LR) systems with a top layer of alkali-developable silicone resist are simple and compatible with conventional photolithography processes. Several chemically amplified resists containing silicon have been proposed.4,5) These resists have utilized the acid-catalyzed condensation of silanol groups in silicone polymer.

Recently, we have also developed a chemically amplified silicone-based negative resist (CSNR) which will be useful for two-layer resist systems. The CSNR is composed of alkali-soluble silicone polymer (ASSP) and an acid generator (AG). Acids are generated from the AG in the $\mathrm{KrF}$ laser exposure area. The acid then catalyzes the condensation reaction of the silanol group in ASSP during post-exposure baking (PEB). This reaction gives the CSNR a negative resist performance. CSNR is alkali-developable and highly transmissible to $\mathrm{KrF}$ excimer laser light. In addition, it shows good resistance to oxygen reactive ion etching $\left(\mathrm{O}_{2} \mathrm{RIE}\right)$. These characteristics are favorable for 
the top imaging layer of two-layer resist systems.

This paper will describe the preparation of the CSNR and its characteristics in KrF excimer laser and electron beam lithographies.

\section{Experimental}

\subsection{Materials}

ASSP was synthesized by means of sol-gel reactions of a mixture of phenyltrimethoxysilane and 2-(3,4-epoxycyclohexyl)ethyl trimethoxysilane purchased from Shin-etsu Chemical Co. Each 0.05 mol of two organotrimethoxysilanes was dissolved in a mixture of $50 \mathrm{ml}$ methanol and $10 \mathrm{ml}$ $1.2 \mathrm{~N}$ aqueous solution of $\mathrm{HCl}$. The solution was refluxed in a flask with a reflux condenser for $1 \mathrm{hr}$. and then some of the methanol was evaporated from the solution at $80{ }^{\circ} \mathrm{C}$. The resulting solution was poured into $500 \mathrm{ml}$ distilled water to obtain a white, sticky ASSP precipitate. The ASSP was dried in a vacuum at $50{ }^{\circ} \mathrm{C}$. Both phenyltrimethoxysilane and 2-(3,4epoxycyclohexyl)ethyl trimethoxysilane are necessary to obtain alkalisoluble silicone polymer. In this experiment, the ASSP was synthesized from an equimolecular solution of two organotrimethoxysilanes. The acid generators (AG's) were from Midori Chemical Co. and they were used without any purification.

The CSNR was prepared by dissolving $1.0 \mathrm{gr}$. of ASSP and $0.03 \mathrm{gr}$. of the acid generator in $7 \mathrm{ml}$ 1-methoxy-2-propanol. Diphenyl (p-thiophenoxy phenyl) sulfonium hexafluoro antimonate was used as the acid generator for $\mathrm{KrF}$ laser evaluation.

\subsection{Characterization}

IR spectra were recorded on a Perkin Elmer Model 1800 spectrometer. ${ }^{29} \mathrm{Si}$ NMR spectra at $79.49 \mathrm{MHz}$ were measured at room temperature with a Bruker MSL-400 spectrometer. Powdered samples were dissolved to a concentration of $30 \mathrm{wt} \%$ in deuterated acetone (acetone- $\mathrm{d}_{6}$ ). The repetition time and spectral width for NMR measurement was $30 \mathrm{~s}$ and $12 \mathrm{kHz}$, respectively. ${ }^{29} 9_{\mathrm{Si}}$ chemical shift was directly read off from internal tetramethylsilane (TMS). Gated decoupling pulse sequence was carried out. GPC measurements were made on a Tosoh Model HLC802UR equipped with four GMHXL-type columns at $40{ }^{\circ} \mathrm{C}$ in THF. The molecular weight (Mw) was determined by polystyrene standards. The silicon content in the ASSP was measured by a neutron activation analysis.

\subsection{Lithographic Evaluation}

A hard baked (at $200{ }^{\circ} \mathrm{C}$ for $60 \mathrm{~min}$ ) OFPR-800 (Tokyo Ohka) was used as the bottom resist in the 2LR application. The CSNR was spun onto the bottom resist layer and prebaked at $100{ }^{\circ} \mathrm{C}$ on a hot plate for $1 \mathrm{~min}$. $\mathrm{KrF}$ exposures were performed using a Nikon model NSR-1755EX8. The numerical aperture value was 0.45 . The exposed resist films were post-exposure baked on a hot plate. Post-exposure bake (PEB) temperature was $85{ }^{\circ} \mathrm{C}$ and the PEB time was $60 \mathrm{sec}$. The exposed and post-exposure baked films were developed in a $1.2 \%$ tetramethylammonium hydroxide (TMAH) aqueous solution for 30 seconds and rinsed for 60 seconds in de-ionized water. The resist thickness was measured with a Dektak 303 profilemeter.

$\mathrm{O}_{2}$ gas reactive ion etching $\left(\mathrm{O}_{2}\right.$ RIE) was carried out with a parallel plate-type reactive ion etcher (ANELVA DEM-451). The conditions were $\mathrm{O}_{2}$ gas flow of $50 \mathrm{sccm}$, gas pressure of $1.3 \mathrm{~Pa}, \mathrm{rf}$ power of $0.1 \mathrm{~W} / \mathrm{cm}^{2}$, and $\mathrm{dc} \mathrm{bias}$ of $500 \mathrm{~V}$. 
3. Results and Discussion

3.1 Solubility of ASSP in alkaline aqueous solutions

Figure 1 shows the IR spectrum of ASSP. Peaks at 3400 and $905 \mathrm{~cm}^{-1}$ are assigned to hydroxy (silanol) groups. It is well known that silanols can be formed by hydrolyzing organomethoxysilane compounds in sol-gel reactions ${ }^{6}$ ). When ASSP is treated with trimethylsilylchloride (TMSCl), these peaks disappear and the solubility of ASSP in alkaline solution is diminished. These results indicate that ASSP is rendered soluble in alkaline aqueous solutions by the presence of silanol groups. A $0.5-\mu \mathrm{m}$-thick ASSP film spin-coated on a silicon wafer was dipped in a $0.9 \mathrm{wt} \%$ TMAH solution and the immersion time to clear the resist film completely was measured. We used this resist-filmclearing time $\left(T_{c}\right)$ as a solubility index. Figure 2 shows the relationship

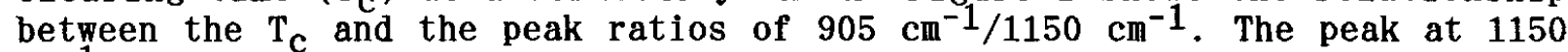
$\mathrm{cm}^{-1}$ is assigned to silicon-phenyl bonds in ASSP. The peak ratios represent silanol contents that strongly depend on the synthetic conditions, that is, the amount of $\mathrm{HCl}$ and the reaction time. $\mathrm{T}_{\mathrm{c}}$ dramatically increased at peak ratios of less than 0.25 . $T_{c}$ at peak ratios of greater than 0.25 was very short, less than 0.5 second. The solubility of the films with peak ratios greater than 0.25 was high enough to apply them to alkali-developable resist materials. We synthesized ASSP with a peak ratio of about 0.3 under the conditions mentioned earlier in the experimental section.

Solubility in alkaline aqueous solution is a desirable characteristic for a matrix resin in multi-component resist materials because it allows development with alkaline aqueous solutions. Conventional novolacdiazonaphthoquinone positive photoresists, which are currently very widely used in VLSI fabrication, use this alkali-development system. Resists which are developed with organic solvents usually swell in the developer, and this swelling reduces pattern resolution. Alkali-developable resists, however, swell little if at all in the developer and therefore exhibit superior performance in terms of resolution characteristics.

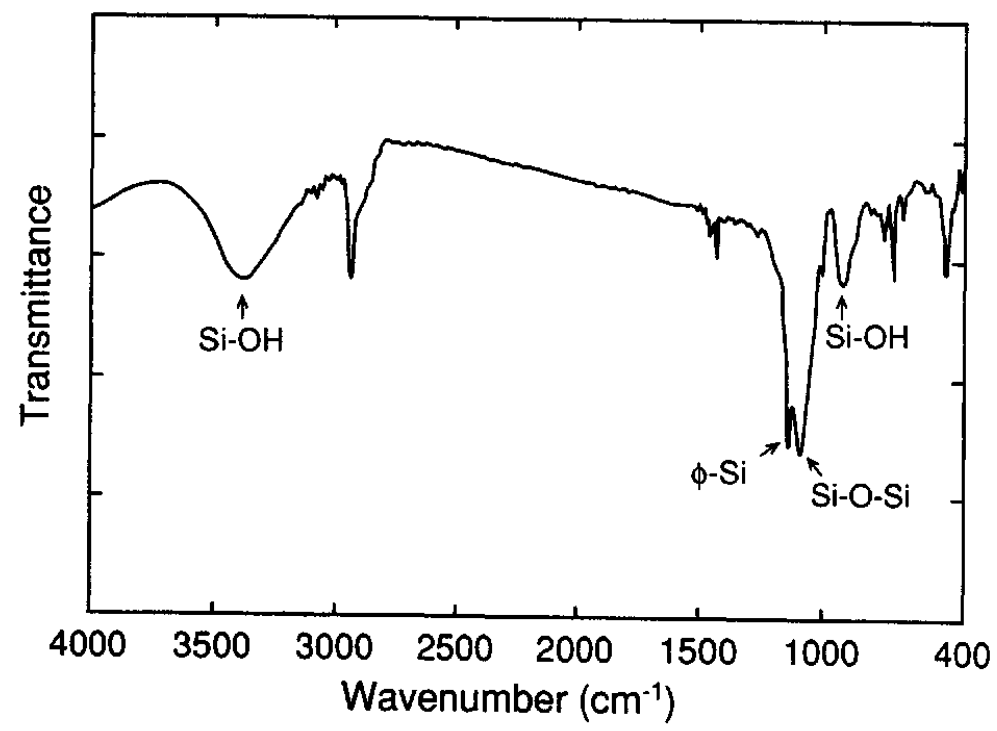

Fig. 1. IR spectrum of alkali-soluble silicone polymer (ASSP). 


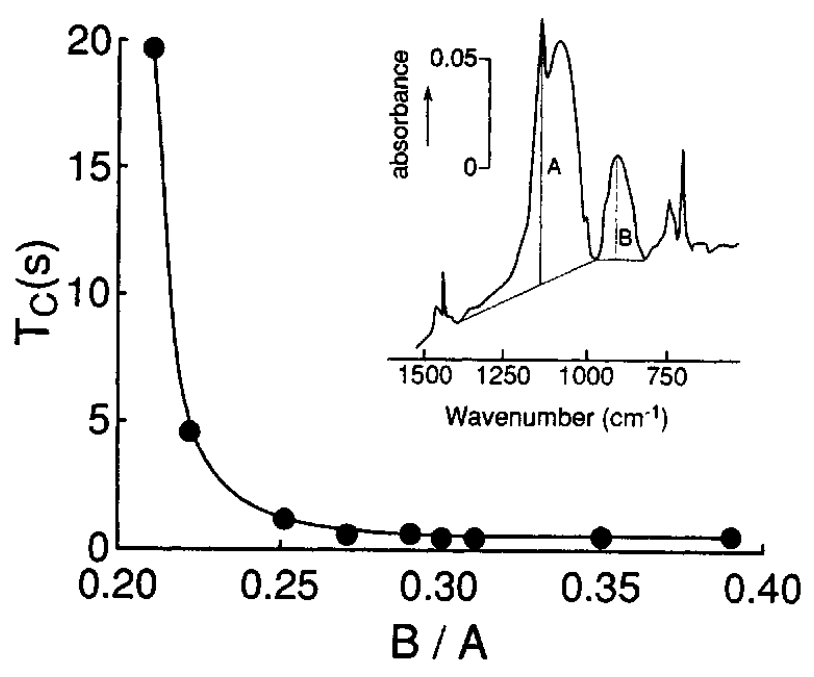

Fig. 2. Relationship between $\mathrm{T}_{\mathrm{c}}$ and peak ratios of $905 \mathrm{~cm}^{-1} / 1150 \mathrm{~cm}^{-1}$.

The presence of silanol groups was also confirmed by ${ }^{29} \mathrm{Si}$ NMR. Figure 3 shows 29 Si NMR spectra of ASSP and silylated ASSP together with assignments of ${ }^{29} \mathrm{Si}$ chemical shifts. ASSP showed two sharp peaks at -70 and at $-57 \mathrm{ppm}$ ((a) in Fig. 3). The former peak is attributed to siloxane units with phenyl and hydroxy side chain groups 7 ), which may condensate to become phenylsilsesquioxane units. The peak of phenyltrimethoxysilane is $-54 \mathrm{ppm}$ and that of

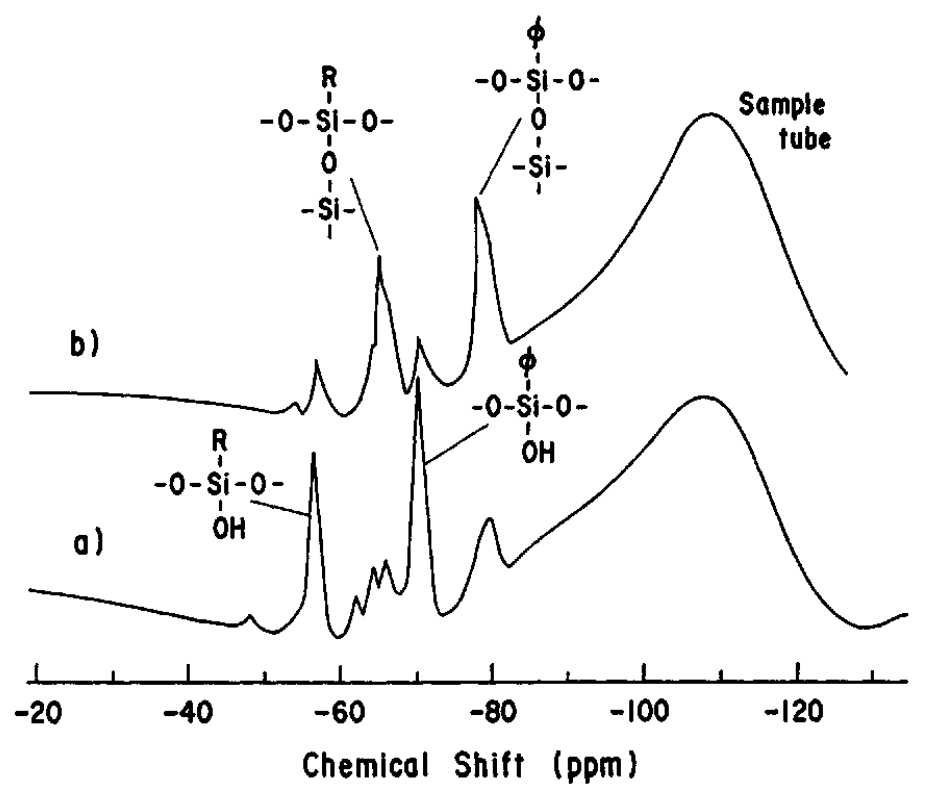

Fig. 3. ${ }^{29}$ Si NMR spectra of ASSP. (a) ASSP before silylation and (b) silylated ASSP. 
2-(3,4-epoxycyclohexyl)ethyl trimethoxysilane is $-41 \mathrm{ppm}$. The difference between the two peaks is $13 \mathrm{ppm}$. This means that the peak at $-57 \mathrm{ppm}$ of ASSP can be attributed to siloxane units with 2-(3,4-epoxycyclohexyl)ethyl and hydroxy side chain groups. When ASSP was treated with trimethylsilylchloride to kill the hydroxyl groups, the peaks at -57 and $-70 \mathrm{ppm}$ became weak and the peaks at -67 and $-80 \mathrm{ppm}$ became strong ((b) in Fig. 3). The strong peak in silylated ASSP is assigned to framework silsesquioxane units. These results indicate that silanol groups are converted to siloxane bonds by silylation.

It is well known that under acidic conditions epoxides may be reacted with water to form glycols. ${ }^{8}$ ) According to neutron activation analysis, the chlorine content in ASSP was about $3.3 \mathrm{wt} \%$. Therefore, we decided that 2(3,4-epoxycyclohexyl)ethyl trimethoxysilane may react with $\mathrm{HCl}$ to form chlorohydrin during this sol-gel reaction as shown for an epoxysilane in eq. (1) below. Chrolohydrin may also play a roll as a promoter of solubility in aqueous solution. In fact, the amount of 2-(3,4-epoxycyclohexyl)ethyl trimethoxysilane was more than $70-\mathrm{mole} \%$ in this sol-gel reaction., so ASSP precipiate could not be obtained from water because ASSP is soluble even in water. This means that a lot of chlorohydrins make the polymer watersoluble.

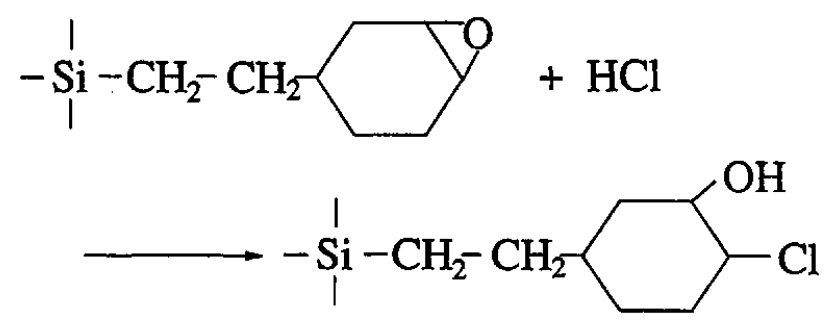

\subsection{Dependence of $\mathrm{HCl}$ concentration on alkali-solubility of ASSP}

The alkali-solubility of ASSP deeply depends on reaction conditions, especially on the concentration of $\mathrm{HCl}$ aqueous solution. Table I shows the relationship between the $\mathrm{HCl}$ concentration and alkali-solubility. When $2.4 \mathrm{~N}$ $\mathrm{HCl}$ solution was used, organosilicon polymer obtained did not dissolve in a $0.9 \mathrm{wt} \%$ TMAH solution during immersion for $60 \mathrm{~s}$. On the other hand, when we used $0.6 \mathrm{~N} \mathrm{HCl}$ solution, ASSP dissolved very rapidly in less than $0.5 \mathrm{~s}$. However, in that case, ASSP precipiate could not be obtained when reaction solution was poured into distilled water. A white emulsion appeared, and then a small white precipitate was obtained from the solution after one day. That is why $1.2 \mathrm{~N} \mathrm{HCl}$ solution was used for synthesis of ASSP as described in the experimental section.

Table I

\begin{tabular}{llllrc} 
PhSi & EPSi & HCl & Mw & yield & solubility \\
\hline $9.9 \mathrm{gr}$ & $12.3 \mathrm{gr}$ & $2.4 \mathrm{~N}$ & 1770 & $14.8 \mathrm{gr}$ & $60 \mathrm{~s}<$ \\
9.9 & 12.3 & 1.2 & 1630 & 11.6 & 0.5 \\
9.9 & 12.3 & 0.6 & 1820 & 4.6 & 0.5 \\
\hline
\end{tabular}

PhSi: Phenyl trimethoxysilane

EPSi: 2-(3,4-epoxycyclohexyl)ethyl trimethoxysilane 
Figure 4 shows the 29Si NMR spectrum of organosilicon polymer obtained from $2.4 \mathrm{~N} \mathrm{HCl} \mathrm{solution.} \mathrm{The} \mathrm{peak} \mathrm{ratios} \mathrm{of} \mathrm{framework} \mathrm{silsesquioxane} \mathrm{units}$ and siloxane units with hydroxy side chain groups are larger in ASSP from $2.4 \mathrm{~N} \mathrm{HCl}$ aqueous solution than from $1.2 \mathrm{~N} \mathrm{HCl}$ solution. This means that silanol groups tend to be converted to siloxane bonds by an excess-HClcatalyzed condensation during a sol-gel reaction. Consequently, the solubility of ASSP from $2.4 \mathrm{~N} \mathrm{HCl}$ solution is worse than from $1.2 \mathrm{~N} \mathrm{HCl}$ solution.

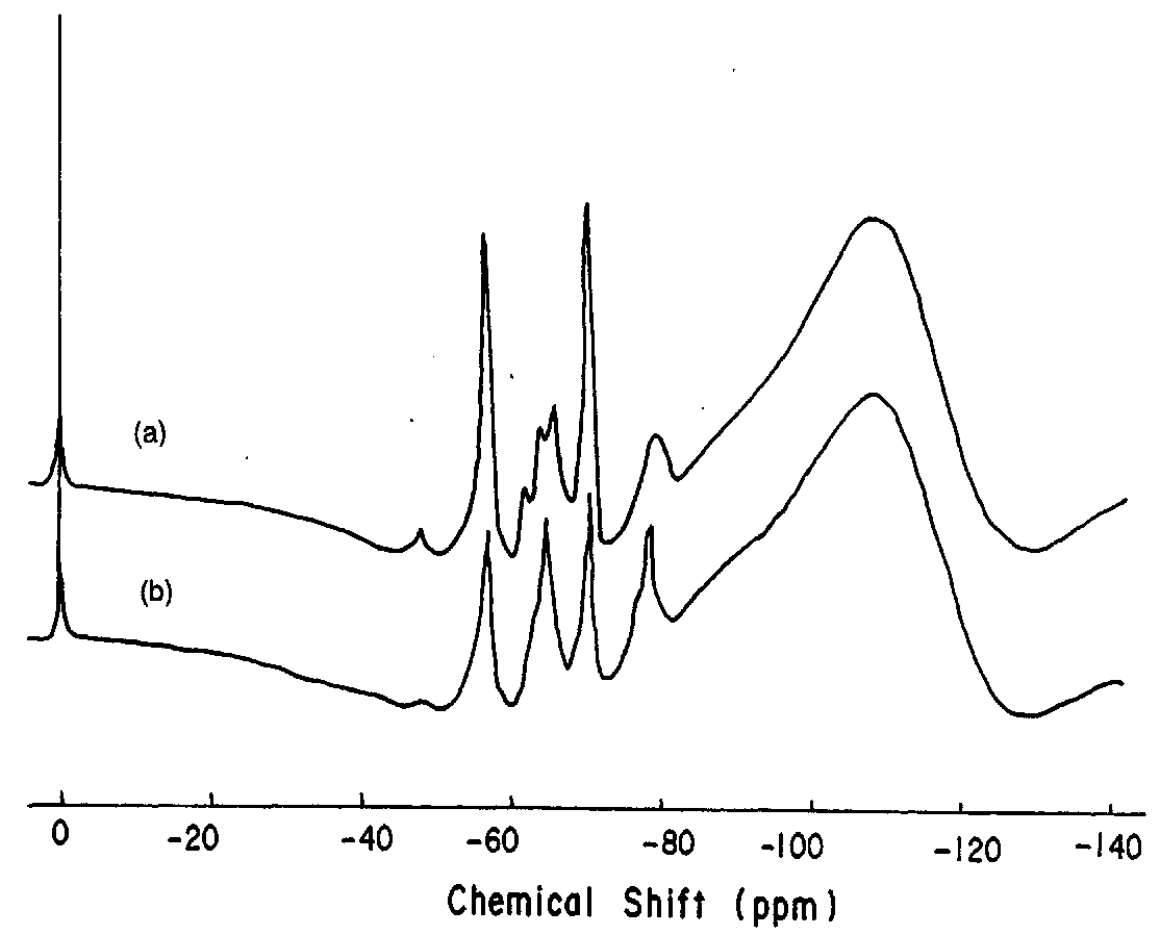

Fig. 4. ${ }^{29} \mathrm{Si}$ NMR spectra of ASSP. (a) $1.2 \mathrm{~N}$ HCl aq. solution, (b) $2.4 \mathrm{~N}$ HCl aq. solution.

\subsection{Chenistry of CSNR}

The resist chemistry of the CSNR was examined by IR and GPC studies. Figure 5 shows the difference IR spectra before and after PEB. The positive peaks indicate the produced species and the negative peaks indicate the diminished species. The exposure dose was $50 \mathrm{~mJ} / \mathrm{cm}^{2}$. The reaction during the PEB process is acid-catalyzed condensation of silanol groups. Figure 5 shows a decrease in silanol absorbances as well as an increase in siloxane absorbance at $1050 \mathrm{~cm}^{-1}$ after PEB ((b) in Fig. 5). Siloxane bonds are formed by the acid-catalyzed condensation of silanol groups, so the alkali-solubility of ASSP was diminished. The solubility of unexposed CSNR films is as high as ASSP. The solubility rate was more than $1000 \mathrm{~nm} / \mathrm{s}$ in a TMAH aqueous solution. On the other hand, CSNR films that were post-exposure baked were only partially developed at about $0.4 \mathrm{~nm} / \mathrm{s}$. The dissolution rate ratio was more than 2500, which is sufficiently high to produce steep profile patterns.

This acid-catalyzed condensation did not occur after $\mathrm{KrF}$ excimer laser exposure and before PEB ((a) in Fig. 5). It has been reported that the condensation reaction of silanol compounds 5,9$)$ occurs in a three-component resist system consisting of novolac resin, diphenylsilanediol, and an AG. 


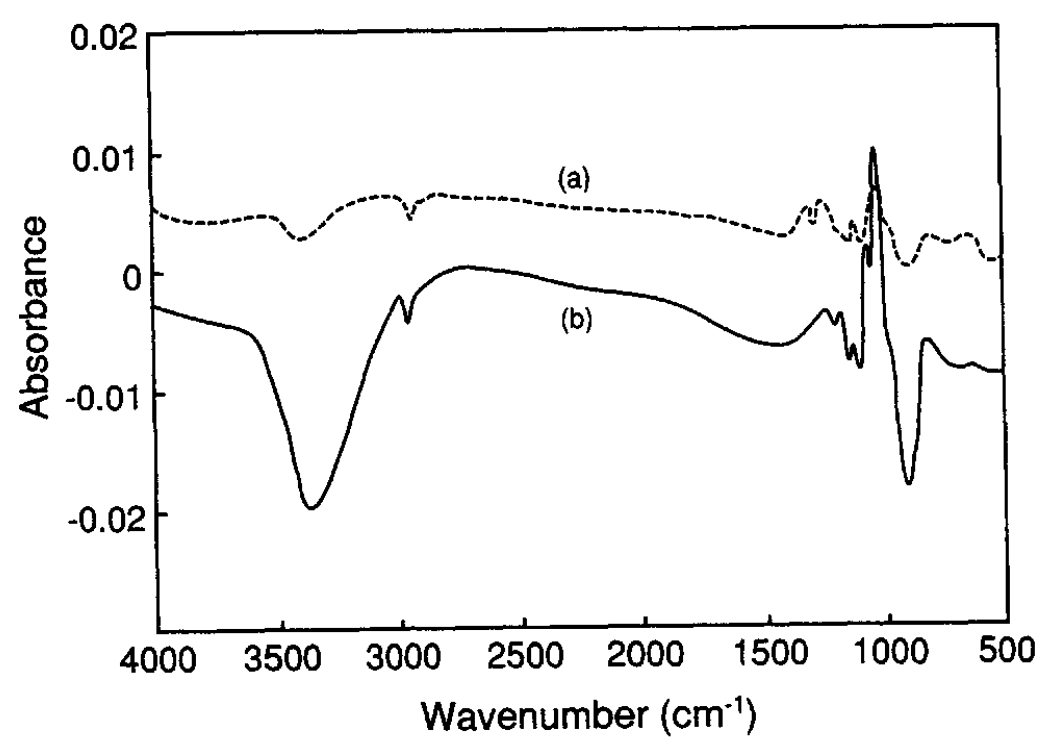

Fig. 5. IR difference spectra of the CSNR, (a) before and (b) after post-exposure bake.

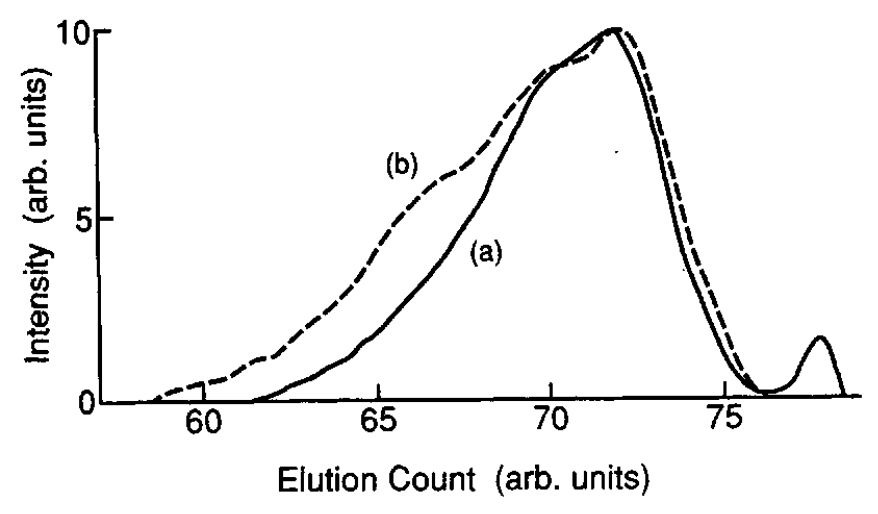

Fig. 6. Molecular distribution curves of the CSNR, (a) before and (b) after post-exposure bake.

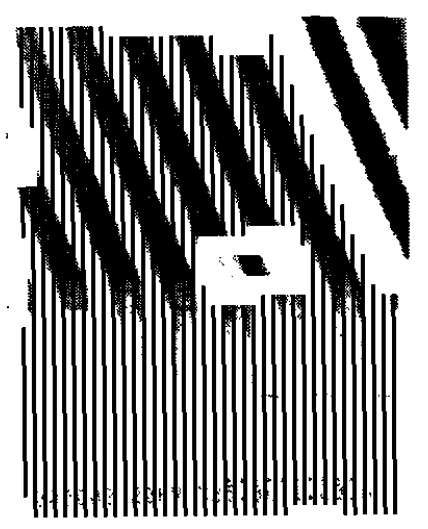

Fig. 7. SEM photograph of $0.25-\mu$. lines-and-spaces patterns fabricated with the CSNR 2LR syster. 
This result is very similar to ours except for the insolubilizing mechanism. The condensation product in the three-component resist system inhibits the dissolution of novolac resin, whereas that in the CSNR becomes insoluble in an alkaline solution itself. This insolubilizing mechanism of CSNR is the same as that of resist materials ${ }^{10}$ ) composed of spin on glass (SOG) and an AG.

The condensation of silanol groups was also confirmed by measuring the molecular weight of the CSNR before and after PEB. Figure 6 shows the molecular distribution changes of the CSNR before and after PEB. The molecular distribution of the CSNR after PEB indicates that it contains a higher number of molecular components than before PEB. The weight average molecular weight of the CSNR was 2030 before PEB and 3300 after PEB, respectively. This change in the molecular weight was due to the condensation reaction of silanol groups in ASSP.

\subsection{0xygen RIE Characteristics}

The CSNR includes silicon atoms so abundant that it exhibits high resistance to $\mathrm{O}_{2}$ RIE. According to a radiation analysis, the silicon content in ASSP was about $17 \mathrm{wt} \%$. The etching rate of the CSNR was less than $5 \mathrm{~nm} / \mathrm{min}$, whereas that of the OFPR- 800 resist was more than $100 \mathrm{~nm} / \mathrm{min}$. The durability ratio of the CSNR to hard baked OFPR- 800 was more than 20 , which is high enough to apply this CSNR to two layer resist systems.

Figure 7 shows $0.25-\mu \mathrm{m}$ lines-and-spaces patterns with a two-layer resist system of CSNR and hard-baked OFPR-800. Fine CSNR patterns were transferred to the bottom layer. The patterns have vertical walls and a thin CSNR layer still remains on top of the bottom-layer patterns, which demonstrates the high resistance of the CSNR to $\mathrm{O}_{2}$ RIE.

\subsection{Sensitivity to an electron beam}

The sensitivity of the CSNR was examined using electron beam exposure. Figure 8 shows its EB sensitivity curves in comparison with five kinds of $A G^{\prime} s$. The sensitivity strongly depends on the $A^{\prime}$ 's. Triphenylsulfonium triflate (TPS-TRF) had the highest sensitivity among the $A G^{\prime}$ 's used in this study. The sensitivity ranking is as follows: Triphenylsulfonium triflate (TPS-TRF) > Triphenylsulfonium hexafluoroantimonate (TPS-ANT) > Bis(tertbutylphenyl)iodonium triflate (BPI-TRF) > Triphenylsulfonium hexafluoroarsenate (TPS-ARS) > Diphenyliodonium hexafluorophosphate (DPI-PHO) >> Tris(chloromethyl)-s-triazine (TCM-TRI). When TCM-TRI, which is not an onium salt, was used as an AG, gelation did not occur after PEB even at an EB exposure dose of more than $100 \mu \mathrm{C} / \mathrm{cm}^{2}$. We have confirmed that TCM-TRI is an effective AG for an acid-hardening three-component resist system composed of novolac resin, melamine compound, and an AG. However, we can not use it for the CSNR. In this system, onium salts were effective for inducing high sensitivity.

Figure 9 shows SEM photographs of delineated $0.3-\mu$ m lines-and-spaces patterns of the CSNR used for the sensitivity evaluation in Fig. 8 . The resolution was compared using CSNR patterns before $0_{2}$ RIE. Although most resist materials exhibit a definite trade-off between sensitivity and resolution, the resist with DPI-PHO had the lowest sensitivity and the lowest resolution among the $A G$ 's shown in Fig. 9. In this CSNR system, therefore, the supposedly inevitable trade-off was not observed. It is well known that the resolution characteristics of chemically amplified resists depend on the diffusion and the reactivity of acids generated by exposure 


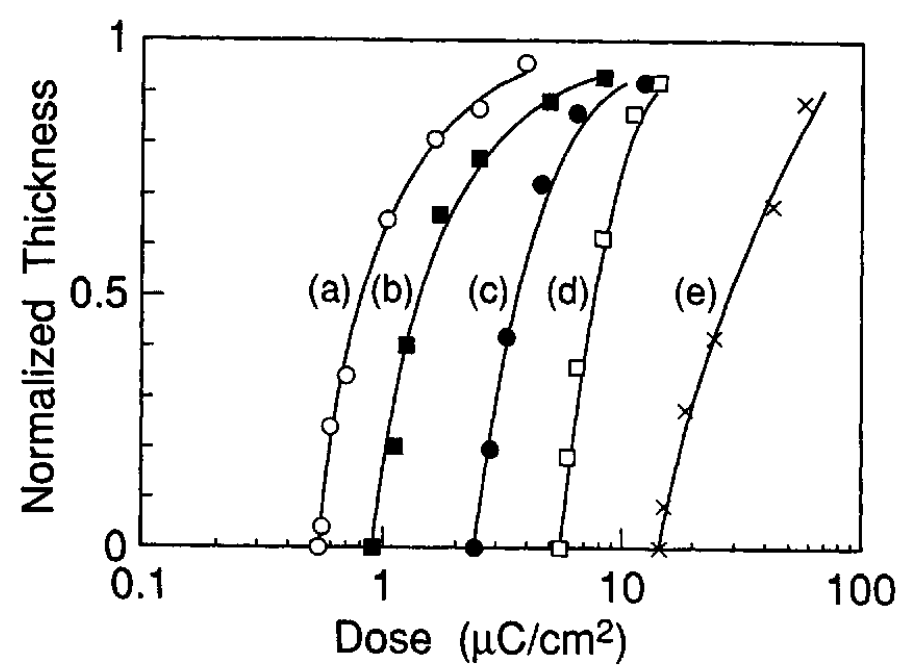

Fig. 8. Electron bean sensitivity of the CSNR. (a) TPS-TRF, (b) TPS-ANT, (c) BPI-TRF, (d) TPS-ARS, (e) DPI-PHO.

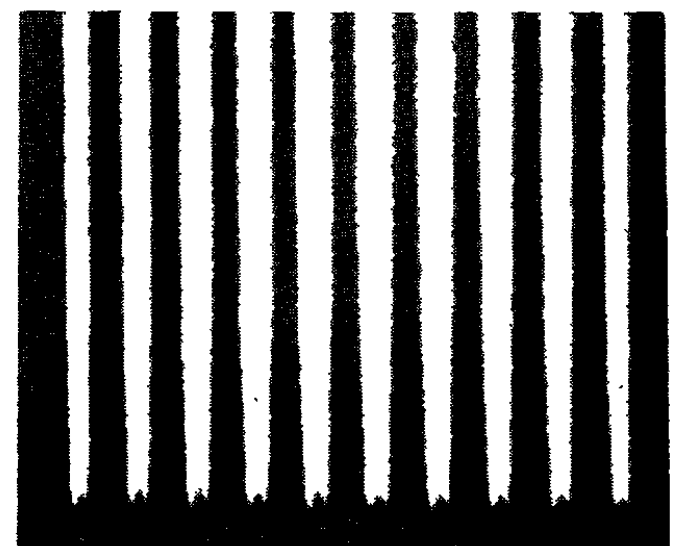

TPS-TRF

$1.2 \mu \mathrm{C} / \mathrm{cm}^{2}$

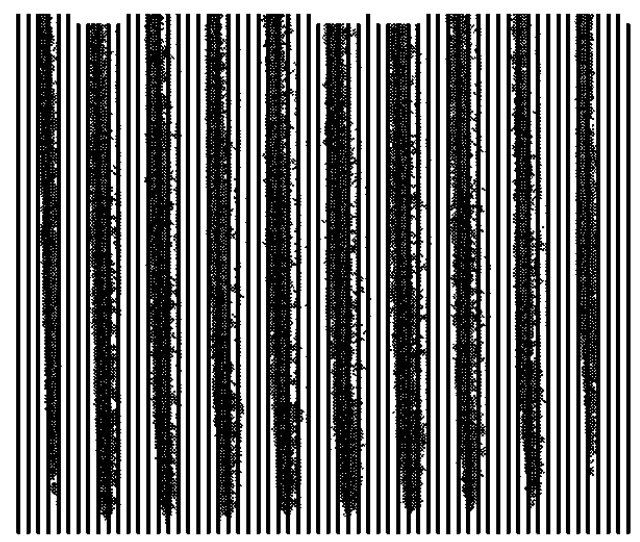

DPI-PHO

$38 \mu \mathrm{C} / \mathrm{cm}^{2}$

Fig. 9. SEM photographs of delineated $0.3-\mu \mathrm{m}$

lines-and-spaces patterns on the bottom layers. 
during $\mathrm{PEB}^{11)}$. Acids from DPI-PHO are supposed to degrade the resolution, and a close investigation of the correlation between resist characteristics and acids from various $A G$ 's is now in progress. Whatever the outcome, it is nevertheless clear that the resist with TPS-TRF exhibits higher sensitivity and resolution than one with DPI-PHO.

\section{Conclusion}

We have developed a chemically amplified silicone-based negative resist composed of an alkali-soluble silicon polymer and an acid generator. The polymer was synthesized through a sol-gel reaction of organotrimethoxysilane. It has a lot of silanol groups, and acids generated in the exposed area catalyze the condensation reaction of these groups, making the polymer insoluble in an alkaline solution. The solubility ratio between exposed and unexposed areas is high enough to fabricate fine patterns. The chemically amplified resist also has high resistance to oxygen RIE. Using a $\mathrm{KrF}$ excimer laser stepper, $0.25-\mu \mathrm{m}$ lines-and-spaces patterns can be fabricated with a two-layer resist system.

The resist's sensitivity to an electron beam depends on the structure of the acid generator. Triphenylsulfonium triflate exhibited the highest sensitivity in this study.

\section{Acknowledgments}

The authors wish to thank Tetsushi Sakai and Tadahito Matsuda for their encouragement and helpful suggestions.

\section{References}

1)H.Ito and C.G.Wilson, ACS Symp. Ser. 242 (1984) 11.

2) J.W.Thackeray, G.W.Orsula, E.K.Pavelchek, D.L.Canistro, L.E.Bogan, Jr., A.K.Berry and K.A.Graziano, Proc. SPIE, 1086 (1989) 34.

3) Omkaram Nalamasu, M.Cheng, J.M.Kometani, S.Vaidya, E.Reichmanis and L.F.Thompson, Proc.SPIE, 1262 (1990) 32.

4)D.R.Mckean, N.J.Clecak and L.A.Pederson, Proc. SPIE, 1262 (1990) 110.

5)T.Ueno, H.Shiraishi, N.Hayashi, K.Tadano, E.Fukuma and T.Iwayanagi, Proc. SPIE, 1262 (1990) 26.

6)L.W.Kelts and N.J.Armstrong, J.Mater.Res., 4 (1989) 423

7) H.Ban,A.Tanaka, and Y.Kawai: Polymer 31 (1990) 564.

8)G.Phillipp and H.Schmidt, J.Non-Crystalline Solids 82 (1986)31.

9) N.Hayashi, K. Tadano, T.Tanaka,H.Shiraishi,T.Ueno, and T.Iwayanagi : Jpn.J.Appl.Phys. 29 (1990) 2632.

10)A.Imai,H.Fukuda,T.Ueno, and S.Okazaki: Jpn.J.Appl.Phys. 29 (1990) 2653.

11) J.Nakamura,H.ban,K.Deguchi, and A.Tanaka: Jpn.J.Appl.Phys. 30 (1991) 2619 . 